\title{
Variety is the spice of life
}

\section{Expanding article contributions to the summer supplement}

The summer supplement issue was originally initiated to provide medical students with an online platform to report unique clinical cases and provide a perspective on a novel topic for their peers. Throughout the past decade, the editors of the UWOMJ have received outstanding submissions that have always provided valuable insight in these Case Report-focused issues. While the summer supplement issue will remain an open forum for clinical cases, this year the UWOMJ editorial board has opened submissions to non-case report articles. We are confident that this decision will provide our authors with an opportunity to expand their scientific reporting skills while contributing more variety in content for our readers.

The online summer supplement issue that you have opened is the first to feature both Case Report and Feature Article submissions. As always, the Case Reports featured in this issue are intriguing cases that are not frequently encountered in the clinic. One article of note is, "Heteropagus twins with hepatobiliary and gastrointestinal connectedness and complex cardiac malformation in autosite" from Teel, Kwan, and Bhaduri. This article is a novel case in the literature that discusses a previously unreported connectedness diagnosis for fetus-in-fetu. The authors review how the methods of diagnosis and the complications of unique connectedness must be considered for physicians moving forward when dealing with these cases.

Updated reviews on a topic of focus are useful tools for providing readers with a summary of the current information available. "Rheumatoid arthritis: a debilitating battle with the human immune system" from Surmanski is one such article that discusses conventional treatments as well as novel therapeutics that are being utilized to improve the quality of life for patients diagnosed with this condition. Feature Articles can also contribute a researched perspective on healthcare initiatives that affect the local community. "Informing ASPIRE and a future student-run clinic: healthcare needs assessment of London, Ontario," by Iacocca et al is one such article that addresses the gaps in the healthcare available to London patients. It posits that this can be alleviated with the launch of the ASPIRE student-run clinic initiative. This initiative can directly impact the surrounding areas that are in critical need of physical and mental health services.

The combination of varying article styles results in different perspectives that maximize the information provided by each type; the first-hand accounts from Case Reports are complemented by the in-depth literature research of Feature Articles. We invite you to join us as we embark on this new chapter for the journal.

Amanda Oakie

Co-Editor-in-Chief 